\title{
The prima donna of epigenetics: the regulation of gene expression by DNA methylation
}

\section{K.F. Santos, \\ T.N. Mazzola and \\ H.F. Carvalho}

Departamento de Biologia Celular, Instituto de Biologia, Universidade Estadual de Campinas, Campinas, SP, Brasil

\section{Correspondence \\ H.F. Carvalho \\ Departamento de Biologia Celular UNICAMP \\ Caixa Postal 6109 \\ 13083-063 Campinas, SP \\ Brasil \\ Fax: +55-19-3788-6111 \\ E-mail: hern@unicamp.br \\ Presented at SIMEC 2004 \\ (International Symposium \\ on Extracellular Matrix), \\ Angra dos Reis, RJ, Brazil, \\ September 27-30, 2004. \\ Research supported by FAPESP, CNPq, FAEPEX-UNICAMP, and CAPES.}

Received February 15, 2005 Accepted July 6, 2005

\begin{abstract}
This review focuses on the mechanisms of DNA methylation, DNA methylation pattern formation and their involvement in gene regulation. Association of DNA methylation with imprinting, embryonic development and human diseases is discussed. Furthermore, besides considering changes in DNA methylation as mechanisms of disease, the role of epigenetics in general and DNA methylation in particular in transgenerational carcinogenesis, in memory formation and behavior establishment are brought about as mechanisms based on the cellular memory of gene expression patterns.
\end{abstract}

Key words

- Epigenetics

- DNA methylation

- Inheritable changes in gene expression

- Transgenerational carcinogenesis

- Environmental influence on memory formation

\section{Introduction}

Cells differ from each other by expressing different combinations of genes at anyone time. The combined product of these genes eventually results in a set of morphological, biochemical and physiological characteristics peculiar to a cell type and its differentiation stage. Transcription factors are crucial determinants in the acquisition and maintenance of the pattern of gene expression shown by cells. They bind to defined DNA sequences at the regulatory region of the genes and trigger events usually associated with chromatin remodeling and activation (or suppression) of gene transcription. However, the number of transcription factors present in a given cell is below the amount necessary to produce enough combinatorial products to define the spectrum of possibilities of gene expression within a genome.

One element contributing to the cells' ability to control wide expression of genes by the genome with a limited number of transcription factors is the stable inactivation of some genes during development or along differentiation within a cell lineage. Control of gene expression based only on transcription factors would imply that gene expression could be reversed by exposure of the cell nucleus to a new set of transcription factors. As a matter of fact, though many aspects of gene expression can be reprogrammed, some marks of differentiation (meaning some expressed or silent genes) are stable so that nuclear transplantation to the cytoplasm of a different cell cannot modify their availability for transcription. These marks are not associated with somatic mutations because there is no modification in DNA sequence during cell differentiation in vertebrates, with the notable exception of sperm cells and lymphocytes (nucleated cells with stable modifications of the genome taking place during differentiation). 
Epigenetics comprises mechanisms of mitotic or meiotic inheritance which are not the consequences of changes in DNA sequence. Epigenetic factors are chemically stable and affect gene transcription, modifying the phenotype with no corresponding changes in the genotype (1). They could suitably explain some differences between monozygotic twins, for example in their susceptibility to diseases (2), even though this hypothesis must take into account environmental effects as modulators or inducers of epigenetic factors. As we shall see later in this review, this is slowly becoming recognized, as more mechanisms and physiological roles of epigenetics are unveiled.

There are at least three epigenetic mechanisms influencing animal development which obeys the inheritability criteria: DNA methylation, histone covalent modification and the Polycomb-trithorax protein complexes.

In spite of the great importance of the latter two and the probable interaction of the three systems within the cell, in this review we shall focus on the mechanisms associated with DNA methylation and their involvement in cell differentiation, development and cancer. The reader may find excellent reviews on histone modification $(1,3)$ and on the Polycomb-trithorax system (4).

\section{DNA methylation}

Since its discovery in 1948, the fifth DNA base - 5-methylcytosine (5-mC) - has generated much controversy with respect to its physiological significance. DNA cytosine methylation is an epigenetic event because a given methylation pattern may be inherited by the daughter cells after a mitotic or meiotic division $(2,5)$. Given its central importance in non-genomic inheritance and earlier discovery, DNA methylation has been named the "prima donna" of epigenetics (6).

DNA methylation in eukaryotic cells involves the addition of a methyl group to the carbon at position 5 of the cytosine ring.
This reaction is catalyzed by the enzyme DNA methyltransferase (DNA-MTase) and this methylation reaction is the most common covalent modification occurring in eukaryotic DNA.

DNA-MTases add methyl groups to cytosine at the $\mathrm{CG}$ position $(\mathrm{CpG}$, with $\mathrm{p}$ corresponding to a phosphate group). The first DNA-MTase gene was cloned from rats and named Dnmt1. This gene is highly conserved among eukaryotic organisms. The mammalian DNA-MTase 1 (DNMT1) has high affinity for a hemimethylated substratum, but is also able to add methyl groups to nonmethylated substrata de novo, thus creating a methylation pattern which differs from that observed in the mother cell $(5,7,8)$. Mutations in the mouse DNMT1 gene lead to an increased deregulation of DNA methylation and gene silencing, and are lethal to the embryo (9). The de novo activity of the mammalian DNMT1 seems to be stimulated by aberrant DNA structures and by $5-\mathrm{mC}$ in only one strand of the DNA.

An evolutionary tendency is the loss of CpG dinucleotides from the genome of higher eukaryotic cells. It is possible that cytosine methylation has played an important role in this trend, because $\mathrm{m}-\mathrm{CpG}$ dinucleotides are easily deaminated to TpG (10), which makes room for gene mutations, especially at hot spots (11).

\section{DNA methylation patterns in animals}

DNA methylation in animals reaches a wide spectrum of levels and patterns. At one extreme is the nematoid Caenorhabiditis elegans, which shows no 5-mC modifications as a result of the derivational loss of a gene coding for the conventional DNAMTase (12). Drosophila melanogaster has a DNA-MTase gene but exhibits low levels of 5-mC modification. The small amount of 5$\mathrm{mC}$ in D. melanogaster appears as $\mathrm{m}-\mathrm{CpT}$ instead of $\mathrm{m}-\mathrm{CpG}$, which occurs in most other organisms. At the other extreme, the 
vertebrate genome has the highest levels of 5 -mC found in the animal kingdom. DNA methylation is dispersed throughout the vertebrate genome in a pattern designated global methylation.

In animal somatic cells, 5-mC represents $1 \%$ of the DNA bases and affects $60-90 \%$ of the $\mathrm{CpG}$ dinucleotides in the genome (13). In the rat, there is a $70 \%$ decrease in the amount of DNA methylation within a short period of the early development. Then de novo methylation recovers the initial levels during implantation. For the rat and probably for other mammals, the cycle of demethylation and de novo methylation is critical for the establishment of the methylation pattern in somatic cells. In mammalian cells in culture, the efficiency in the maintenance of the methylation pattern during cell division is between 97 and 99.9\%. De novo methylation rates are about 3-5\% per mitosis. Although very low, this de novo methylation might cause modification of the epigenetic markers $(2,14)$.

The most striking characteristic of the DNA methylation patterns in vertebrates is the presence of $\mathrm{CpG}$ islands (non-methylated CpG-rich regions) (15). Computer analysis of the human genome has revealed the presence of about 29,000 CpG islands (16) and has shown that about $60 \%$ of the human genes have associated $\mathrm{CpG}$ islands, most of which remain non-methylated during development in most tissues (17). Of this total, $70 \%$ are associated with human genes and over half of them are located at the 5'-end of genes and present a potential regulatory function through DNA methylation (18).

A primary effect of the environment on DNA methylation patterns results from the fact that the presence in the diet of methyl group donors and co-factors, such as the amino acid methionine, which are necessary for the methylation reaction, influences the overall methylation levels (19).

The methylation and demethylation reactions during germ cell differentiation and then soon after fecundation are extensively involved in imprinting mechanisms. Imprinting refers to the selective inactivation by DNA methylation in addition to other factors of a parent specific allele. The setting of imprinting takes place by a methylation wave occurring soon after fecundation. The pattern of parental imprinting is erased very early during germ cell development so that alleles coming from the parent of the opposite sex will then be set to behave according to the sex of the individual. Details of imprinting are found in many textbooks and a good overview is provided by Lewin's Genes VIII (20), including mechanisms involved in normal and abnormal imprinting of $I G F$-2 (insulin-like growth factor II gene) and its membrane receptor (IGF2R), which are perhaps the most studied imprinted genes. Besides details on imprinting, Hartl and Jones (21) provide a discussion on DNA methylation-dependent co-suppression mechanisms in plants that might be of interest to plant biologists.

Lists of both human (cancer.otago.ac.nz/ IGC/Web/home.html) and mouse (www. mgu.har.mrc.ac.uk/research/imprinting/) imprinted genes are available in the World Wide Web.

\section{The origin of the $\mathrm{CpG}$ islands}

As mentioned before, a $\mathrm{CpG}$ island is a DNA sequence with a high incidence of CpG dinucleotides which remains unmethylated after development or in differentiated cells. A major issue in epigenetics is the question of how such islands resist methylation.

The binding of a protein to the DNA would sterically hinder the binding of a DNAMTase. If such protein was present at the time of de novo methylation, then it could contribute to the maintenance of that sequence as a non-methylated $\mathrm{CpG}$ island. However, this possibility remains elusive and such blocking protein has not been identified as yet. A second possibility for the 
maintenance of these non-methylated islands would be the recognition of given chromatin structures achieved by specific patterns of histone covalent modifications (see below).

It is also possible that $\mathrm{CpG}$ islands appear by demethylation. Such demethylation activity could take place through a thermodynamically unfavorable break in the carbon-carbon bond joining the pyrimidine to its methyl group or by a repair process through the excision of the methylated 5-mC and the addition of a cytosine. In plants, a DNA glycosylase enzyme, named DEMETER, removes the methyl groups of $\mathrm{m}-\mathrm{CpG}$ (22). However, there is no animal counterpart to this enzyme, a fact that weakens this hypothesis.

A small, though important, part of the $\mathrm{CpG}$ islands becomes methylated during development and, when this occurs, the gene becomes stably silent. The programmed methylation of the $\mathrm{CpG}$ islands during development is involved in the imprinting of the genome and inactivation of the $\mathrm{X}$ chromosome (see below). De novo methylation occurs in the embryonic germ cells, suggesting high activity in this lineage, though somatic cells are also thought to be subjected to methylation. A given fraction of the human $\mathrm{CpG}$ islands undergoes progressive methylation after development in some tissues and in cancer cells.

\section{Origin of the methylation patterns, their maintenance and loss}

The mechanisms underlying the establishment of a given methylation pattern are still unknown. However, it is already recognized that the DNA-MTases DNMT3A and DNMT3B are responsible for new methylation of the DNA (de novo methyltransferases). These enzymes are highly expressed in young embryonic cells, during the period when de novo methylation takes place. They are essential for the embryonic development of mice and mutations in the corresponding human genes lead to mental retardation, atypical craniofacial development and instability of the repetitive pericentromeric DNA (5). How these enzymes identify the genome regions to be methylated in each cell type is still unknown.

One possibility is that the de novo methylation occurring in mammalian cells at the beginning of development does not discriminate the target genes, but will affect all available CpG. This default (or global) methylation is compatible with the fact that there is no sequence in the mammalian genomes which are intrinsically non-methylated. Even some CpG islands, which most of the time are non-methylated, may be methylated under certain circumstances during normal or tumoral development. It is also clear that not every region of the genome is equally accessible to the DNA-MTases. Particularly, DNMT3B is known to be required for the de novo methylation of specific genome regions such as the repetitive pericentromeric DNA and $\mathrm{CpG}$ islands in the inactive $\mathrm{X}$ chromosome, indicating a preferential activity towards silent chromatin regions.

Evidence corroborating the idea that accessory factors are required for the proper methylation arose from plants. In these organisms, an SNF-2-like chromatin remodeling factor is essential for the complete methylation of the Arabidopsis thaliana genome (23). Thus, one may assume that DNA methylation requires a disturbance of the chromatin structure by these remodeling factors, allowing the access of DNA-MTases to the DNA. This sequence of events seems to be particularly necessary for the activation of genes in heterochromatic regions.

Another hypothesis to explain global methylation is the fact that the methylation machinery of mammals acts preferentially on certain DNA sequences, particularly the repetitive ones. The presence of high levels of methylation at certain regions would function as a methylation center, thus spreading the methylation to adjacent regions. Barriers 
to this spread would result in the formation of $\mathrm{CpG}$ islands. A probable starting factor for this methylation would be some DNA repetitive sequences, even though it is not known whether they correspond to direct or indirect causes of methylation. The clearest definition of a methylation center came from studies on Neurospora (24).

New findings on the methylation of DNA came from studies on the post-transcriptional silencing of genes in plants. Doublestrand RNA triggers the destruction of cognate transcripts and is the basis for the widely diffused siRNA technology. There is a suggestion that this process results in the methylation of the corresponding DNA in the genome. The post-transcriptional silencing of genes through double-strand RNA is an ancient mechanism of protection of the genome, occurring in fungi, plants and animals. However, these two processes are not obligatorily related to each other, since silencing is present in $C$. elegans in the complete absence of 5-mC. The clear association between double-strand RNA and gene silencing through DNA methylation still requires further investigation.

The maintenance of methylation patterns depends on mechanisms that reproduce a given methylation pattern along cell generations. A conceivable mechanism results from the semi-conservative duplication of the methylation pattern followed by the activity of DNMT1, which adds methyl groups to the new strand at the points where the parental strand bears m-CpG(25). Through this mechanism, the pattern of methylation (i.e., methylated and non-methylated sequences) is copied and the epigenetic information is transferred along cell generations and also to the organism's next generation. In plants, the enzyme DNA methyltransferase 1 (MET1), ortholog to the animal DMT1, exhibits the same activity and consequently is responsible for the imprinting observed in these organisms (22).

The idea that new methylation patterns are established at the beginning of development by the DNMT3A and DNMT3B de novo methyl transferase and then reproduced in the somatic cells by the DNMT1 seems appealing but cannot explain the preservation of methylation patterns during cell proliferation. It was reported that cultured tumor cells lacking DNMT1 activity $\left(\mathrm{DNMT1}^{-/-}\right.$) showed only a $20 \%$ reduction in the level of genomic methylation during culturing, as compared to normal cells. Though detailed patterns of methylation are not preserved at the level of single $\mathrm{CpG}$, the status of methylation of DNA domains seems to be propagated during development with high efficiency. CpG islands preserve their methylated or non-methylated state in an extremely stable manner for several cell generations. DNMT1 is partially responsible for this stability, but it is likely that additional factors are involved in the preservation of the methylation status of individual $\mathrm{CpG}$ islands, since, as mentioned above, methylation patterns are preserved even in the absence of the only known maintenance methyl transferase, DNMT1 (5).

There is still much debate about the possibility that loss of methylation would affect genome integrity, as represented by bizarre chromosomal rearrangements due to nonhomologous recombination and higher mutation incidence in genes found in hypomethylated regions. New experimental evidence will certainly direct this discussion. For instance, deletion of DNMT3 resulted in chromosomal instability and spontaneous immortalization of mouse embryonic fibroblasts (26), reinforcing a role of DNA methylation in genome integrity.

\section{Gene silencing}

DNA methylation causes changes in chromatin structure, modifying the interactions between the DNA and activating or repressing transcription factors (or complexes).

Could one assume that transcriptional 
inactivity would result in DNA methylation? Studies on the mechanisms for the maintenance of $\mathrm{CpG}$ islands in a non-methylated state give support to this idea. The coincidence between the location of $\mathrm{CpG}$ islands and promotor regions is astonishing. Further coincidence between the 5'-end of the $\mathrm{CpG}$ island and the transcription factor binding region is usually observed. The potential importance of the promoter activity and the genesis of the $\mathrm{CpG}$ island was demonstrated by studies with transgenic mice. Transgenes containing $\mathrm{CpG}$ islands usually reproduce the non-methylated status, but their resistance to methylation is lost if the promoter activity is below normal, so that transcriptional inactivity may lead to de novo methylation.

It is not clear at present whether there is some sort of signal from an inactive gene that would result in silencing through DNA methylation. One attractive possibility is that chromatin structure may be informative to the methylation machinery. Lysine acetylation at the histone tails by histone acetyl transferases facilitates the access of transcription factors to the gene. Histone deacetylases (HDAC) reverse the process, reducing the transcription rate of the gene (27). It is worth mentioning at this point that mutations in the HDAC genes may result in cancer (1). This implies that the covalent modifications of the core histones are intimately associated with the transcriptional activity and could be read by the methylation machinery. Studies in Neurospora, Drosophila and other organisms have indicated a clear association between histone methylation and DNA methylation, as particularly shown by the demonstration that inactivating mutation in the gene of a histone methyltransferase [with activity towards Lys9 of histone H3 (H3K9)] abolished genome methylation. In mammals and in yeast, Lys9 methylation in histone $\mathrm{H} 3$ is associated with transcriptionally repressed heterochromatin. If DNA methylation in mammals is proven to be dependent on histone methylation, the idea that DNA methylation targets previously silenced genes will gain further support (28).

Jackson and colleagues (29), studying DNA methylation in A. thaliana, observed three possible methylation forms of Lys9 of histone H3 (monomethylated, dimethylated and trimethylated). Each form results from the activity of the methyl transferase kryptonite. Kryptonite mutations resulted in a reduced rate of methylated $\mathrm{CpNpG}(\mathrm{N}$ meaning any base) and suppression of the silencing of genes SUPERMANn, TA3 and FWA, besides the reactivation of transposable elements. These findings suggest that $\mathrm{H} 3 \mathrm{~K} 9$ methylation is associated with DNA methylation also in plants. However, while $\mathrm{H} 3 \mathrm{~K} 9$ methylation is required for methylation of all CpG sites in Neurospora, it is necessary only for the methylation of $\mathrm{CpNpG}$ sites in Arabidopsis.

Methylation of a $\mathrm{CpG}$ sequence at the promoter region causes the binding of proteins containing the methylated CpG-binding domain (MBD) and transcription suppressors, such as HDAC, blocking the beginning of transcription in mammals. In $\mathrm{Neu}$ rospora, methylation may also interrupt transcription of a transcriptionally active gene (15). Four of the five MBD already identified show transcription inhibition activity, establishing complexes with HDAC, nucleosome remodeling proteins and transcription repressors (30). MeCP2, an MBD, shows affinity for hypermethylated DNA sequences and may act by recruiting a transcription repressor protein complex $(1,5)$.

Non-methylated transgenes, retrotransposons and repetitive DNA may have a disordered expression and could contribute to genome destruction, suggesting that DNA methylation has a defensive or protective function (31).

Methylation of a previously silenced gene would cause an irrevocable suppression. Methylation clearly contributes to the stability 
of the inactivation, in events such as inactivation of the $\mathrm{X}$ chromosome and retroviral genes. On the other hand, demethylation agents may reverse the methylation status of the genome. Genes present in the inactive $\mathrm{X}$ chromosome are reactivated in rat embryonic cells lacking DNA-MTases. Treatment with 5-azacytidine, a demethylating agent, also interferes with the imprinting observed during $\mathrm{X}$ chromosome inactivation. 5-Azacytidine is a potential antineoplastic drug when hypermethylation is detected as a causative agent of tumor growth (32).

In addition to the above mentioned possibility that, besides degradation of the corresponding mRNA, double-strand RNA introduction in a cell would also suppress the expression of the corresponding gene through DNA methylation, it was recently shown that genes located downstream to the estrogen receptor activation, such as the progesterone receptor, become stably inactivated and suppressed by DNA methylation in the absence of estrogen stimulation (33). This has a profound influence on mammary gland cancer because later reintroduction of estrogen is not sufficient to reestablish expression of the progesterone receptor gene.

\section{DNA methylation and diseases}

Unusual methylation or demethylation does affect the human phenotype, giving rise to several syndromes and diseases. Many diseases are now better understood in the light of epigenetics, especially after the consideration of the methylation status of genes and genome. Centromere instability, facial abnormalities, Rett syndrome, immunodeficiency, autoimmunity, and neoplasias may originate from abnormal methylation during or after development (15).

Tzao and colleagues (34) investigated the possible mechanisms involved in the changes occurring in the fragile histone triad gene (FHIT), a putative tumor suppressor gene, in the genesis of lung neoplasias. FHIT modifications are frequently found in lung tumors. Besides, mutations in this gene are also found in bronchial lesions in chronic smokers, suggesting that FHIT deregulation anticipates tumor development, appearing very early during carcinogenesis. Fifty percent of the patients with lung cancer showed reduced FHIT protein, with a significant correlation between the abnormal expression of protein and alternative splicing in $\mathrm{CpG}$ islands at the 5'-end of the gene. This reinforces the idea that DNA methylation may be of diagnostic potential in lung and other cancers.

The study of Oelke and colleagues (35) showed that DNA hypomethylation contributes to changes in gene expression and function of T lymphocytes in systemic lupus erythematosus. Treatment of $\mathrm{T}$ cells of normal donors with 5-azacytidine led to the identification of methylation-sensitive genes. The co-stimulatory molecule CD70, whose overexpression results in the production of polyclonal IgG in lupus patients, was identified as one of the important genes. $\mathrm{T}$ cells treated with different demethylation drugs resulted in increased CD70 mRNA and protein levels and caused an increase in the secretion of IgG. This latter effect was reversed by treatment with anti-CD70 antibodies. Despite these observations, the demethylated sequences responsible for the increased expression of CD70 have not been identified.

DNA methylation has been implicated in a series of hematological diseases and efforts for the development of epigenetic drugs and therapies are growing and will shortly be successful in some areas $(32,36)$.

\section{Abnormal DNA methylation in cancer: hypermethylation or hypomethylation?}

Promoter hypermethylation has been pointed out as an important phenomenon during cancer development (28). Methylation of 
sequences corresponding to genes coding for components of the DNA repair system and tumor suppressor genes is usual in different tumors (37), and its importance in tumor development is equivalent to that of gene mutation and loss of heterozygosity (38).

A first assumption regarding the effect of DNA hypomethylation is gene activation. As a matter of fact, H-RAS, C-MYC, CT genes (genes normally expressed in the testis and aberrantly activated in tumors), MAGE in melanoma and CAGE in testis cancer are activated by hypomethylation. The gene products resulting from gene activation by DNA hypomethylation correspond to cyclin D2 and maspin in gastric carcinoma, MN/CA9 in renal cell carcinoma, S100A4 in colon cancer metastatic cells, and in $14-3-3 \sigma$ (among other proliferationassociated proteins) in pancreatic cancer (36).

A second event resulting from DNA hypomethylation in tumors is chromosomal instability. De-repression of satellite sequences may result in non-homologous recombination and chromosomal alterations (6). However, the chromosomal instability appearing in tumors and related to hypomethylation is not global, but specific, resulting in typical chromosomal rearrangements, the most commonly found being $t(1,6)$ usually associated with Wilm's tumor (39).

On the other hand, targets of hypermethylation are inactivated. To develop tumors, these targets must be negative regulators of cell cycle progression or positive regulators of resistance to apoptosis. As a matter of fact, the tumor suppressor genes $P 16, V H L$, $R B, A P C$, and BRCAl were found to be silenced by DNA hypermethylation in one or more tumors $(36,40)$. Hypermethylation of the $\mathrm{CpG}$ islands in the promoter region of tumor suppressor genes has been proposed as a diagnosing tool for lung and colorectal neoplasias (30).

However, since most of the information on DNA hypomethylation in cancer results from the total amount of $\mathrm{m}-\mathrm{CpG}$, which may not directly represent demethylation of regulatory $\mathrm{m}-\mathrm{CpG}$, and despite the knowledge of hypomethylation target genes in tumors, there is a prevalent idea that hypermethylation and hypomethylation are not mutually exclusive in cancer.

As mentioned above, imprinting is an epigenetic mechanism that distinguishes alleles whose sequences are identical but that were provided by each parent. The adaptive advantage of imprinting genes is not currently clear, though several hypotheses exist in attempts to explain it (41). Loss of imprinting (LOI) implies biallelic expression or suppression of genes that should be imprinted. Wilm's tumor is attributed to biallelic expression of $I G F-2$ and suppression of $H 19$. The former seems to increase resistance to apoptosis and the latter seems to result in loss of growth suppressor elements. LOI may occur in somatic as well as in germ cells. Patients with germinal LOI exhibit the Beckwith-Wiedmann syndrome and a higher incidence of tumors, with 1000-fold the frequency of non-affected individual.

LOI in chromosomes $19 q$ and $9 p$ are related to oligodendrogliomas and childhood acute lymphoblastic leukemias, respectively (36).

Transgenerational carcinogenesis represents an additional step in the complexity of the involvement of DNA methylation in cancer development. Transgenerational carcinogenesis corresponds to the transmission of tumor susceptibility to the progeny by parents exposed to carcinogens before mating. In an experimental model (42), exposure to chromium (III) affected DNA methylation in sperm cells, with increased hypomethylation effects on the $45 \mathrm{~S}$ ribosomal genes. The treatment also resulted in a progeny of individuals larger than the parents and with increased T3 levels. Microarray analysis of the liver in the progeny revealed that genes involved in tumor growth/suppression were affected, potentially increasing the possibility of tumor development. 
Though appearing as a proposed mechanism and based only on a few experimental models, the inheritability of environmentally induced epigenetic markers must be considered as an appealing possibility to explain the high incidence of cancer in these individuals that cannot be attributed solely to germ cell mutation (43).

\section{Memories: remembering Mom's sweet embrace}

Epigenetic mechanisms have been identified in neuron development and synapse formation. Neuronal exclusive expression of neuron-specific genes is controlled by epigenetic mechanism mainly associated with histone modification. RE-1 silencing transcription factor (REST) is a transcription factor which binds to and blocks a neuronrestrictive silencer element found in the promoter region of neuron-specific genes. REST expression and function blocks the expression of neuron-specific genes in non-neuronal cells. After binding to the neuronrestrictive silencer element, REST recruits co-factors, histone acetylases and histone methyltransferases, resulting in overall reorganization of the local chromatin (44). Involvement of DNA methylation in this process has not been characterized as yet. Since histone covalent modifications as an epigenetic mechanism are not the focus of the present review, we will direct the reader to a recent review on the epigenetic mechanisms controlling memory formation, human cognition development and impairment in some neurodegenerative diseases (45) and concentrate the discussion on other aspects of neurobiology in which DNA methylation plays a controversial central role.

Schizophrenia is a serious disorder resulting in patient inability to deal with daily social situations and difficulties in performing simple cognitive tasks. The etiology of schizophrenia has been correlated with deficiency of an extracellular matrix protein named reelin by a growing body of evidence (46). Inhibitors of either HDAC or DNMTs increase reelin expression, indicating their regulation by histone acetylation and DNA methylation $(46,47)$.

DNA methylation and associated control have been implicated in Rett's syndrome, a disease responsible for most of the intellectual disabilities observed in girls. This syndrome results from germline mutations in $\mathrm{MeCP} 2$ and the disease is suggestively associated with the derepression of genes normally suppressed by methylation. Since there is no general gene derepression, it is thought that MeCP2 might function in the control of specific genes associated with neural development and function (48).

Rat behavior and response to stress have long been known to be modulated by aspects of maternal guarding during the first week of lactation. This effect is not dependent on the genome, because foster mothers elicit a less fearful and more modest hypothalamic-pituitary-adrenal response to stress in their progeny with licking and grooming activities (49). Weaver and colleagues (50) have mapped this phenomenon to the expression levels of the glucocorticoid receptor gene in the hippocampus and have demonstrated that DNA methylation and other factors are involved in the epigenetic regulation of the expression levels of the glucocorticoid receptor gene. They also observed that the phenomenon is affected by central infusion of a histone deacetylase inhibitor.

A general conclusion based on the present review is that epigenetics is an important adaptive factor allowing the offspring to adapt to subtle changes in the environment detected through modifications in parental behavior.

It is worth stressing that DNA methylation appears to be an epigenetic mechanism allowing the perpetuation of an acquired behavior across generations and that de novo methylation occurs during the early postnatal period. Since the only genes currently known to be de 
novo methylated during the postnatal period are the glucocorticoid receptor gene mentioned above and the Hoxa5 and Hoxb5 genes (51), it remains to be determined whether this is a general or specific event.

It is tempting to ask whether imprinting at the molecular level would correlate with androgen and/or estrogen imprinting in the hypothalamus which also occurs during the first days after birth and controls sexual behavior after puberty in rats (52-54).

We conclude that the primordial function of de novo methylation is the generation of a memory of the gene expression pattern established in embryos throughout later development and adulthood, including definition of imprinting patterns. An emerging function is the physiological role in keeping the structural stability of chromosomes and their normal behavior during mitotic and meiotic cell divisions.

The molecular mechanisms generating the methylation patterns are still poorly understood. Uncertain are also the mechanisms preserving the $\mathrm{CpG}$ island in a nonmethylated state and how the rest of the DNA is methylated.

However, increasing knowledge about the current involvement of epigenetics in general, and DNA methylation in particular, in human diseases has brought such phenomena to center stage in biomedical research. It became clear that methylation is not a primary event in gene silencing, but usually takes place in genes previously repressed by other mechanisms. Besides, it is possible that different epigenetic mechanisms act in concert at different developmental stages, defining the memory of complex patterns of gene expression.

Finally, we can predict that the importance of events regulated by DNA methylation and the development of tools such as methylation-sensitive representational difference analysis $(55,56)$ and epigenetic drugs $(32,57)$ will certainly encourage the study of the epigenome.

\section{Acknowledgments}

The authors are grateful to Christine Hackel and Márcio Lorencini for useful comments on the manuscript. The authors apologize to researchers involved in the primary discoveries discussed in this review who were not cited because of space limitation.

\section{References}

1. Cho KS, Elizondo LI \& Boerkoel CF (2004). Advances in chromatin remodeling and human disease. Current Opinion in Genetics and Development, 14: 308-315.

2. Vercelli D (2004). Genetics, epigenetics, and the environment: switching, buffering, releasing. Journal of Allergy and Clinical Immunology, 113: 381-386.

3. Peterson CL \& Laniel MA (2004). Histones and histone modifications. Current Biology, 4: R546-R551.

4. Ringrose L \& Paro R (2004). Epigenetic regulation of cellular memory by the Polycomb and Trithorax group proteins. Annual Review of Genetics, 38: 413-443.

5. Urnov FD \& Wolffe AP (2001). Above and within the genome: epigenetics past and present. Journal of Mammary Gland Biology and Neoplasia, 6: 153-167.

6. Scarano MI, Strazzullo M, Matarazzo MR et al. (2005). DNA methylation 40 years later: Its role in human health and disease. Journal of Cellular Physiology, 204: 21-35.

7. Vertino PM, Yen RW, Gao J et al. (1996). De novo methylation of $\mathrm{CpG}$ island sequences in human fibroblasts overexpressing DNA (cytosine-5-)-methyltransferase. Molecular and Cellular Biology, 16:
4555-4565.

8. Ponger L \& Li-W-H (2005). Evolutionary diversification of DNA methyltransferases in eukaryotic genomes. Molecular Biology and Evolution, 22: 1119-1128.

9. Li E, Bestor TH \& Jaenisch R (2001). Targeted mutation of the DNA methyltransferase gene results in embryonic lethality. Cell, 69: 915926.

10. Scarano E (1973). DNA methylation. Nature, 246: 539 (Letter).

11. Murata J, Tada M, Iggo RD et al. (1997). Nitric oxide as a carcinogen: Analysis by yeast functional assay of inactivating p53 mutations induced by nitric oxide. Mutation Research, 379: 211-218.

12. Gutierrez A \& Sommer RJ (2004). Evolution of dnmt-2 and mbd-2like genes in the free-living nematodes Pristionchus pacificus, Caenorhabditis elegans and Caenorhabditis briggsae. Nucleic Acids Research, 32: 6388-6396.

13. Nakao M (2001). Epigenetics: interaction of DNA methylation and chromatin. Gene, 278: 25-31.

14. Bird A (2002). DNA methylation patterns and epigenetic memory. Genes and Development, 16: 6-21.

15. Jones PA \& Takai D (2001). The role of DNA methylation in mamma- 
lian epigenetics. Science, 293: 1068-1070.

16. Lander ES, Linton LM, Birren B et al. (2001). Initial sequencing and analysis of the human genome. Nature, 409: 860-921.

17. Yamashita R, Suzuki $Y$, Sugano $S$ et al. (2005). Genome-wide analysis reveals strong correlation between $\mathrm{CpG}$ islands with nearby transcription start sites of genes and their tissue specificity. Gene, 350: 129-136.

18. Wang $Y$ \& Leug FC (2004). An evaluation of new criteria for CpG islands in the human genome as gene markers. Bioinformatics, 20: 1170-1177.

19. Selhub J \& Miller JW (1992). The pathogenesis of homocysteinemia: interruption of the coordinate regulation by $S$-adenosylmethionine of the remethylation and transsulfuration of homocysteine. American Journal of Clinical Nutrition, 55: 131-138.

20. Lewin B (2004). Genes VIII. Pearson Education, Upper Saddle River, NJ, USA.

21. Hartl DL \& Jones EW (2001). Genetics - Analysis of Genes and Genomes. 5th edn. Jones \& Bartlett Publishers, London, UK.

22. Scott RJ \& Spielman M (2004). Epigenetics: imprinting in plants and mammals - the same but different? Current Biology, 14: R201-203.

23. Brzeski J \& Jerzmanowski A (2002). Deficient in DNA methylation 1 (DDM1) defines a novel family of chromatin-remodeling factors. Journal of Biological Chemistry, 278: 823-828.

24. Miao VP, Freitag M \& Selker EU (2000). Short TpA-rich segments of the zeta-eta region induce DNA methylation in Neurospora crassa. Journal of Molecular Biology, 300: 249-273.

25. Vertino PM, Sekowski JA, Coll JM et al. (2002). DNMT1 is a component of a multiprotein DNA replication complex. Cell Cycle, 1: 416-423.

26. Dodge JE, Okano M, Dick F et al. (2005). Inactivation of Dnmt3b in mouse embryonic fibroblasts results in DNA hypomethylation, chromosomal instability, and spontaneous immortalization. Journal of Biological Chemistry, 280: 17986-17991.

27. Wade PA (2001). Transcriptional control at regulatory checkpoints by histone deacetylases: molecular connections between cancer and chromatin. Human Molecular Genetics, 10: 693-698.

28. Jones PA (2002). DNA methylation and cancer. Oncogene, 21: 5358-5360.

29. Jackson JP, Johnson L, Jasencakova $Z$ et al. (2004). Dimethylation of histone $\mathrm{H} 3$ lysine 9 is a critical mark for DNA methylation and gene silencing in Arabidopsis thaliana. Chromosoma, 112: 308-315.

30. Belinsky SA (2002). Epigenetics is alive and growing. Trends in Molecular Medicine, 8: 53-54.

31. Bestor TH (2000). The DNA methyltransferases of mammals. Human Molecular Genetics, 9: 2395-2402.

32. Egger G, Liang G, Aparicio A et al. (2004). Epigenetics in human disease and prospects for epigenetic therapy. Nature, 429: 457-463.

33. Leu YW, Yan PS, Fan M et al. (2004). Loss of estrogen receptor signaling triggers epigenetic silencing of downstream targets in breast cancer. Cancer Research, 64: 8184-8192.

34. Tzao C, Tsai H, Chen J et al. (2004). 5'CpG island hypermethylation and aberrant transcript splicing both contribute to the inactivation of the FHIT gene in resected non-small cell lung cancer. European Journal of Cancer, 40: 2175-2183.

35. Oelke K, Lu Q, Richardson D et al. (2004). Overexpression of CD70 and overstimulation of IgG synthesis by lupus $T$ cells and $T$ cells treated with DNA methylation inhibitor. Arthritis and Rheumatism, 50: $1850-1860$.

36. Feinberg AP \& Tycko B (2004). The history of cancer epigenetics. Nature Reviews. Cancer, 4: 143-153.

37. Esteller M (2005). Dormant hypermethylated tumour suppressor genes: questions and answers. Journal of Pathology, 205: 172-180.
38. Jones PA \& Laird PW (1999). Cancer epigenetics comes of age. Nature Genetics, 21: 163-167.

39. Qu GZ, Grundy PE, Narayan A et al. (1999). Frequent hypomethylation in Wilm's tumors of pericentromeric DNA in chromosomes 1 and 16. Cancer Genetics and Cytogenetics, 109: 34-39.

40. Esteller M (2003). Cancer epigenetics: DNA methylation and chromatin alterations in human cancer. Advances in Experimental Medicine and Biology, 532: 39-49.

41. Wrzeska M \& Rejduch B (2004). Genomic imprinting in mammals. Journal of Applied Genetics, 45: 427-433.

42. Cheng RY, Hockman T, Crawford E et al. (2004). Epigenetic and gene expression changes related to transgenerational carcinogenesis. Molecular Carcinogenesis, 40: 1-11.

43. Anderson LM, Diwan BA, Fear NT et al. (2000). Critical windows of exposure for children's health: cancer in human epidemiological studies and neoplasms in experimental animal models. Environmental Health Perspectives, 108 (Suppl 3): 573-594.

44. Huang $Y$, Myers SJ \& Dingledine R (1999). Transcriptional repression by REST: recruitment of $\operatorname{Sin} 3 \mathrm{~A}$ and histone deacetylase to neuronal genes. Nature Neurosciences, 2: 867-872.

45. Levenson JM \& Sweatt JD (2005). Epigenetic mechanisms in memory formation. Nature Reviews. Neuroscience, 6: 108-118.

46. Costa E, Chen Y, Davis J et al. (2002). REELIN and schizophrenia: a disease at the interface of the genome and the epigenome. Molecular Intervention, 2: 47-57.

47. Chen Y, Sharma RP, Costa RH et al. (2002). On the epigenetic regulation of the human reelin promoter. Nucleic Acids Research, 30: 2930-2939.

48. Chen WG, Chang Q, Lin Y et al. (2003). Derepression of BDNF transcription involves calcium-dependent phosphorylation of MeCP2. Science, 302: 885-889.

49. Francis D, Diorio J, Liu D et al. (1999). Nongenomic transmission across generations of maternal behavior and stress responses in the rat. Science, 286: 1155-1158.

50. Weaver IC, Cervoni N, Champagne FA et al. (2004). Epigenetic programming by maternal behavior. Nature Neurosciences, 7: 847854.

51. Hershko AY, Kafri T, Fainsod A et al. (2003). Methylation of HoxA5 and HoxB5 and its relevance to expression during mouse development. Gene, 302: 65-72.

52. Dohler KD (1991). The pre and postnatal influence of hormones and neurotransmitters on sexual differentiation of the mammalian hypothalamus. International Review of Cytology, 131: 1-57.

53. MacLusky NJ \& Naftolin F (1981). Sexual differentiation of the central nervous system. Science, 211: 1294-1303.

54. Piffer RC \& Pereira OC (2004). Reproductive aspects in female rats exposed prenatally to hydrocortisone. Comparative Biochemistry and Physiology. Part C, Pharmacology, Toxicology and Endocrinology, 139: 11-16.

55. Ushijima T, Morimura K, Hosoya Y et al. (1997). Establishment of methylation-sensitive-representational difference analysis and isolation of hypo- and hypermethylated genomic fragments in mouse liver tumors. Proceedings of the National Academy of Sciences, USA, 94: 2284-2289.

56. Kaneda A, Takai D, Kaminishi M et al. (2003). Methylation-sensitive representational difference analysis and its application to cancer research. Proceedings of the National Academy Sciences, USA, 983: $131-141$

57. Ushijima T \& Okochi-Takada E (2005). Aberrant methylations in cancer cells: Where do they come from? Cancer Science, 96: 206211. 